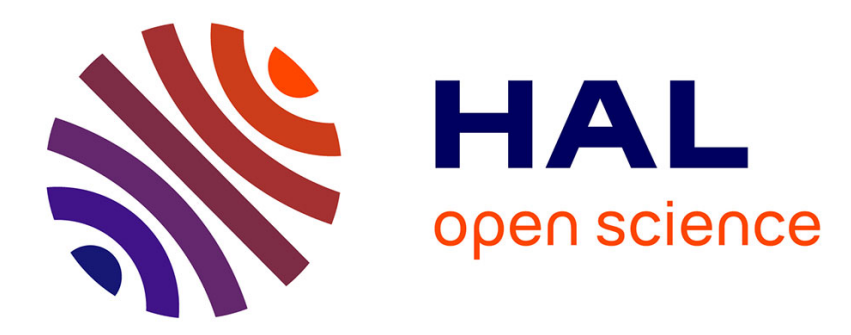

\title{
Inégalités de genre et approche par les capabilités : quelle mise en dialogue chez Sen?
}

\author{
Muriel Gilardone
}

\section{To cite this version:}

Muriel Gilardone. Inégalités de genre et approche par les capabilités: quelle mise en dialogue chez Sen?. Revue Tiers Monde, 2009, 198 (198), pp.357-371. halshs-00419370

\section{HAL Id: halshs-00419370 \\ https://shs.hal.science/halshs-00419370}

Submitted on 7 Dec 2018

HAL is a multi-disciplinary open access archive for the deposit and dissemination of scientific research documents, whether they are published or not. The documents may come from teaching and research institutions in France or abroad, or from public or private research centers.
L'archive ouverte pluridisciplinaire HAL, est destinée au dépôt et à la diffusion de documents scientifiques de niveau recherche, publiés ou non, émanant des établissements d'enseignement et de recherche français ou étrangers, des laboratoires publics ou privés. 


\title{
INÉGALITÉS DE GENRE ET APPROCHE PAR LES CAPABILITÉS : QUELLE MISE EN DIALOGUE CHEZ SEN?
}

\author{
Muriel GILARDONE *
}

\begin{abstract}
Quelle relation y a-t-il entre l'intérêt d'Amartya SEN pour la question des inégalités de genre et l'élaboration de son approche par les capabilités? De nombreux arguments de cette approche puisent indiscutablement leur force d'une perspective qui tient compte du désavantage relatif des femmes - pour SEN, l'une des inégalités les plus criantes. Il est donc instructif, et peut-être même crucial, de revenir sur la démarche scientifique qui est au fondement de sa vision du genre et des inégalités sexuées. En particulier, nous montrerons comment l'étude de la répartition alimentaire au sein de la famille lui permet elle d'aboutir à une comprébension plus claire des enjeux de la capabilité individuelle.
\end{abstract}

Mots clés : Amartya SEN, capabilité individuelle, inégalités de genre, genre, répartition alimentaire

L'intérêt de SEN pour les inégalités de genre apparait au début des années 1980 avec la publication d'articles spécifiquement dédiés à ce sujet. Ses recherches se font principalement à trois niveaux : le mode de répartition de la nourriture au sein de la famille, la surmortalité féminine, et les sources d'inégalité entre hommes et femmes (I). Bien que ses travaux aient un caractère empirique et local - SEN s'intéressant plus particulièrement à la situation des femmes indiennes - il en a tiré des leçons importantes pour les fondements théoriques des études de la pauvreté, des inégalités et, plus généralement, de l'avantage humain. L'observation des attitudes au sein de la sphère familiale et de leurs conséquences sur des facteurs objectifs du bien-être révèle notamment le rôle clé des «préférences adaptatives » dans la perpétuation des inégalités et, en définitive, l'incapacité des critères fondés sur l'utilité à saisir le désavantage relatif des femmes (II).

\footnotetext{
* Centre de recherche en économie et management, CREM, Université de Caen Basse-Normandie.
} 
Confortant ses critiques du welfarisme ${ }^{1}$ et du ressourcisme ${ }^{2}$, il défend plus ardemment encore son approche par les capabilités de base et complète son argumentation en faisant appel à la distinction mise en évidence par I. BERLIN (1969) entre « liberté positive » et « liberté négative» (III). Son approche englobe et dépasse la conception de la liberté négative - qui, prise isolément rejoint la problématique libertarienne - et tente de saisir l'ensemble des conditions de son exercice en intégrant dans l'analyse des caractéristiques personnelles et sociales. En particulier, «le genre, en tant que paramètre essentiel de l'analyse économique et sociale, est complémentaire, et non concurrent, des variables relatives à la classe sociale, à la propriété, à la profession, au revenu et au statut familial » (SEN, 1990, p. 129). Les implications pratiques de son approche par les capabilités, que ce soit en termes d'évaluation des états sociaux ou en termes de politiques à mettre en œuvre, sont complexes et loin d'être claires, mais SEN estime que leur portée peut être immense, notamment en ce qui concerne les inégalités persistantes comme celles que représentent les inégalités de genre.

\section{I - DES DROIT D'ACCÈS FAMILIAUX AUX FONCTIONNEMENTS INDIVIDUELS}

Dans «Family and Food: Sex Bias in Poverty», SEN (1984a) complète son analyse de la consommation de nourriture des individus (SEN, 1981) en précisant qu'au-delà du pouvoir de commande d'une famille sur les biens alimentaires, il faut tenir compte de la répartition de ces biens au sein même de la famille. En effet, «les droits d'accès des familles ne déterminent pas ce qu'un membre particulier de la famille peut manger» (Ibid., p. 346). Il lui a donc semblé pertinent de s'intéresser à la répartition de la nourriture dans les familles, qui devient dès lors plus une question de conventions et de mours que de droits légaux. SEN souligne plus particulièrement la présence de «biais sexué » dans la répartition alimentaire intrafamiliale, ce qui l'amène à considérer le genre comme une variable fondamentale de l'accès individuel à la nourriture, et donc une variable fondamentale de la capabilité de base à bien se nourrir.

La difficulté de ce sujet tient à l'obtention de données précises concernant qui mange quoi dans une famille. Cette question est généralement considérée comme «faisant partie de la vie privée d'une famille, et il est peu probable qu'un observateur puisse venir et mesurer précisément ce qui se passe, sans affecter le phénomène observé» (Ibid., p. 347). L'idée qu'il existe un biais sexué contre les femmes et les filles dans la répartition intrafamiliale des biens alimentaires peut donc sembler difficile à prouver. Pourtant un certain nombre d'études ont été

1 - SEN (1979) a employé le terme «welfarisme» pour désigner cette conception longtemps exclusive de l'économie normative : « l'économie du bien-être » où les utilités individuelles étaient les seuls matériaux pertinents pour définir la justice des états sociaux.

2 - Le « ressourcisme» se distingue du welfarisme en refusant de mesurer l'avantage individuel avec une mesure aussi subjective que l'utilité, et en préférant des mesures plus objectives comme l'accès à certaines ressources. 
menées en ce sens dans différents pays ${ }^{3}$, apportant des preuves de cette inégalité. SEN, lui, se concentre sur la région du Bengale, tout en estimant que ses analyses peuvent avoir une pertinence générale pour la compréhension de la pauvreté et de la malnutrition dans le Tiers-Monde.

Afin d'apporter des preuves du biais sexué en faveur des hommes dans la répartition intrafamiliale de nourriture dans cette région spécifique, SEN (1984a, p. 348) mobilise des données récoltées par l'Institut des sciences de l'alimentation et de la nutrition de l'Université de Dakha (INFS, 1977) ainsi que celles de chercheurs du Centre international de recherche sur les maladies diarrhéiques (Chen, HuQ et D'SouzA, 1980). Elles concernent les consommations de calories et de protéines des hommes et des femmes de différentes classes d'âge observées dans un large échantillon de familles issues de différents villages du Bangladesh, en 1975-1976, et durant l'été 1978. Les observations montrent que les membres féminins de chaque classe d'âge consomment moins de calories et moins de protéines que leurs homologues masculins, la différence s'accentuant au-delà de 45 ans et de 16 à 19 ans. Toutefois, ces données n'ont pas permis de conclure à l'existence d'un biais systématique en faveur des hommes dans la répartition familiale de nourriture :

La difficulté repose non seulement sur les doutes possibles quant à la nature représentative des deux échantillons, mais aussi sur le fait que les soi-disant «besoins » alimentaires pouvaient être différents pour les hommes et pour les femmes. En effet, l'étude de l'Institut postule des besoins alimentaires qui ont pour effet de montrer que les hommes sont plus désavantagés que les femmes vis-à-vis de la relation entre la nourriture consommée et les «besoins » (Ibid., p. 349).

Or, SEN se montre très sceptique concernant les chiffres représentant les besoins nutritionnels postulés par l'étude, fondés sur les recommandations de l'OMS et de la FAO en 1973. Non seulement de nombreuses études ont critiqué la base de ces calculs, mais SEN estime qu'il y a de bonnes raisons de remettre en cause les hypothèses relatives aux activités féminines, "qui ne sont pas aussi « sédentaires » que les calculs de calories tendent à le supposer» (Ibid., p. 351). À cela, SEN ajoute deux autres failles des calculs : 1) la non prise en compte des besoins nutritionnels supplémentaires des femmes enceintes et de celles qui allaitent ; 2) le risque de raisonnement circulaire des calculs reliant les besoins caloriques aux caractéristiques physiques, négligeant le fait que le poids d'une personne ainsi que son niveau d'activité dépendent essentiellement de sa consommation alimentaire. En ce sens, SEN semble considérer ce type de données comme une preuve de l'accès défavorisé des femmes à la nourriture au sein des familles, réduisant leur capabilité à bien se nourrir.

Ce n'est toutefois pas la seule donnée pertinente. En effet, il cite une étude du contexte socio-économique et des conditions de santé à Calcutta entre 1976 et 1978 (CMDA, 1980), s'intéressant plus particulièrement à la morbidité respective des hommes et des femmes. Or, à quelques exceptions près, la morbidité féminine atteint un niveau bien plus élevé que la morbidité masculine dans toutes

3 - SEn (1984a, pp. 347-348) recense diverses études qui, depuis les années 1940, ont prouvé que le phénomène était avéré en Afrique, en Asie, en Amérique du Sud et même en dehors du Tiers-Monde, en Écosse et aux États-Unis notamment pendant les périodes de guerre ou de dépression. 
les classes d'âge. SEN (Ibid., p. 356) estime que l'on peut en tirer des conclusions quant à la malnutrition des femmes :

Il n'est bien sûr en aucun cas évident que la morbidité soit d'abord le résultat de la malnutrition. Mais la malnutrition est l'un des facteurs de morbidité, et les caractéristiques de la privation de nourriture peuvent bien aller de pair avec d'autres types de privation. Dans tous les cas, [...] l'image de la morbidité - donnant vraisemblablement une preuve indirecte de disparités nutritionnelles - indique une discrimination contre les femmes.

Cependant, ces données se fondent sur des entretiens et sont inévitablement discutables. Mais, pour SEN (Ibid., n.b.p.), si elles doivent être prises avec précaution, c'est surtout en raison de la tendance féminine à sous-évaluer sa morbidité, ce qui renforce la démonstration.

En collaboration avec J. KYNCH, SEN (1983) complète son étude de la situation des femmes en Inde en mobilisant d'autres données statistiques. L'analyse des consommations alimentaires étant difficile à réaliser au sein des familles, il se concentre sur «les capabilités en termes de longévité, de santé, de nutrition, etc. qui posent moins de problèmes d'observation» (Ibid., p. 367). Les données du recensement indien entre 1901 et 1981 révèlent une caractéristique démographique frappante de l'Inde : le déclin constant du ratio femme/homme. En effet, en 1901, ce ratio est de 0,972 et il n'atteint plus que 0,935 en 1981. L'étude plus précise de ce ratio par tranches d'âge prouve en outre que la faiblesse relative de la population féminine en Inde ne s'explique pas par une différence de ratio à la naissance - ce ratio étant favorable aux femmes de 0 à 34 ans. Dès lors, le problème est celui d'un différentiel de mortalité : l'Inde apparaît comme l'un des rares pays au monde où l'espérance de vie à la naissance est plus faible pour les femmes que pour les hommes.

Ce constat contredit l'idée répandue selon laquelle le biais contre les femmes diminue avec le progrès économique. En Inde, le déclin à la fois du ratio de la population féminine par rapport à la population masculine et de l'espérance de vie des femmes est allé de pair avec un déclin du taux de mortalité global. Les explications fréquemment retenues sont les suivantes : 1) une préférence pour les garçons menant à une négligence des bébés filles; 2) certains types de mortalité sont sélectifs entre les hommes et les femmes ; 3) une mortalité en couche élevée (PADMANABHA, 1981, p. 35). La première explication intuitive citée n'est souvent pas considérée sérieusement, car difficile à prouver. Pourtant, les études sur la consommation alimentaire des filles et des garçons, citées par SEN dans la publication précédemment étudiée, semblent attester d'une négligence réelle. En outre, SEN a lui-même cherché à apporter une contribution empirique permettant d'augmenter les preuves en ce sens. Entre janvier et mars 1983, il collecte avec S. SENGUPTA des données concernant des caractéristiques physiques des garçons et des filles âgés de 0 à 5 ans dans deux villages du Bengale Ouest :

nous avons trouvé un biais sexué considérable contre les filles dans l'un des villages (Kuchli) et un autre plus faible [...] dans l'autre village (Sahajapur), distant de seulement dix kilomètres du premier. L'analyse causale des facteurs potentiellement déterminants indiquait plusieurs paramètres pertinents. [...] À Kuchli, les politiques de réforme agraire ont eu bien plus de succès, avec pour résultat que seulement $18 \%$ des enfants 
appartiennent désormais à des familles sans terre, alors qu'ils sont 60 \% à Sahajapur. Le niveau nutritionnel des enfants est en conséquence plus élevé à Kuchli, mais en même temps l'étendue du biais sexué [...] est bien plus élevée. En fait, la position nutritionnelle des filles dans les deux villages est à peu près la même, et à la fois le niveau moyen de nutrition et le plus grand différentiel sexué à Kuchli sont largement le résultat de la même différence, à savoir le niveau plus élevé de nutrition des garçons à Kuchli qu'à Sahajapur. (SEN, 1983, p. 23)

Dès lors, il est tentant de conclure que les améliorations économiques bénéficient aux garçons et n'améliorent pas la situation des filles. Mais, la différence entre les deux villages s'explique par un autre facteur : à Sahajapur, un programme d'aide alimentaire direct avait été mis en place pour les populations les plus pauvres, réduisant considérablement le différentiel nutritionnel entre les filles et les garçons bénéficiaires de ce programme. Cette étude permet donc à SEN (1983, p. 24) de justifier son intérêt pour la répartition intrafamiliale dans les problèmes d'inégalité :

Bien que les réformes agraires fournissent [...] une base plus solide pour la prospérité générale, leur incapacité à fournir une proposition honnête aux filles vient des biais au sein du processus familial d'allocation, qui répartit les effets de la réforme agraire. Les interventions nutritives directes, d'un autre côté, réduisent le rôle du chef de famille et des autres membres adultes de la famille, ce qui peut réduire le biais contre les filles.

Une fois le biais sexué dans la répartition intrafamiliale de nourriture identifié, en saisir les racines n'est pas chose aisée. KYNCH et SEN (1983, p. 370) distinguent cependant deux types de raisonnement qui sont autant d'hypothèses à tester. Le premier est relatif à la pertinence des considérations sociales, comme l'importance de la progéniture mâle pour la «perpétuation» de la famille dans des sociétés dominées par les hommes. Le second est fondé sur les facteurs économiques, tels que 1) l'attente d'un soutien de la part des fils et non des filles durant la vieillesse, 2) l'attente d'un soutien financier ou d'une aide pour les activités agricoles de la part des fils et de leurs épouses et non des filles qui à l'âge adulte quittent le foyer, ou même 3) la portée plus grande du travail agricole des petits garçons par rapport à celui des petites filles. Toutefois, si le deuxième type de raisonnement oriente vers des causes tout à fait probables, SEN met en garde contre une conclusion hâtive qui amènerait à confirmer un modèle beckerien de comportement individuel cherchant à maximiser l'utilité sans égard pour les contraintes sociales. Il voit au moins deux facteurs qui impliquent un biais sexué en aucun cas fondé sur un calcul des gains et des pertes d'utilité. D'une part, les attentes des familles peuvent créer un «biais dans la perception » des besoins et des mérites respectifs des garçons et des filles. D'autre part, la mère d'un bébé garçon reçoit généralement plus d'attention, et donc mange plus que le reste de la famille, que la mère d'un bébé fille, ce qui crée un biais sexué indirect transitant par l'allaitement, et non par une répartition observable de nourriture en défaveur des filles.

Ces articles de SEN ont surtout une préoccupation empirique et leur contenu analytique est assez faible. Cependant, SEN ne perd pas de vue les implications théoriques que suggèrent ces études, et qui apparaissent implicitement dans le choix des données statistiques traitées. En effet, les données utilisées font référence à des fonctionnements - comme le fait d'être bien nourri ou en bonne santé 
- et à des capabilités - comme la capabilité de vivre longtemps -, et non à une notion subjective du bien-être ou au pouvoir légal de commande sur les biens. De manière ponctuelle, SEN annonce que ces études peuvent avoir une portée importante au niveau de la théorie économique liée à l'économie du bien-être, aux statistiques normatives, et à la planification (SEN, 1984a, p. 346). Ce sont principalement ces implications qui nous intéresseront maintenant.

\section{II - LES PRÉFÉRENCES ADAPTATIVES : UNE LIMITE CONSIDÉRABLE DU « WELFARISME »}

La famille constitue le plus souvent l'unité où se prennent les décisions de travail et de consommation. Dès lors, si l'on observe une discrimination systématique envers certains membres, il devient difficile de relier ces décisions au bien-être individuel. À cet égard, SEN (1984a, pp. 360-362, nous soulignons) cherche à remettre en cause au moins trois tendances de la théorie économique traditionnelle:

L'une est simplement d'abstraire la famille, et de continuer l'analyse comme si chaque individu prenait les décisions de lui-même, ce qui est la structure typique de, disons, la littérature théorique sur l'«équilibre général». [...] La seconde approche est d'ignorer carrément les individus, et de prendre la famille comme unité d'analyse - des décisions, des actions, et même du bien-être [...] Il serait très curieux que la vision du bien-être de la famille du chef de famille soit tout ce qui compte [...] La troisième approche consiste à postuler une harmonie complète au sein de la famille, le bien-être de chacun des membres étant également servi par les décisions familiales [...].

Les analyses de SEN montrent que l'on ne peut ni abstraire l'individu de la famille, ni postuler une répartition harmonieuse du bien-être parmi les membres d'une même famille. SEN (Ibid.) fait d'ailleurs une distinction entre le fait de considérer le bien-être individuel comme «indépendant» vis-à-vis du reste de la famille et le fait que l'on puisse considérer «séparément» le bien-être des différents membres d'une famille. Il refuse la première approche, mais estime indispensable la seconde, arguant que « les bien-être individuels peuvent être interdépendants, mais distincts» (Ibid.). En revanche, il affirme que la notion de bienêtre elle-même doit être repensée. Il lui semble clair qu'une notion « introspective » du bien-être individuel n'est pas soutenable, en particulier en ce qui concerne les nourrissons et les enfants, mais également envers certains membres adultes de la famille qui peuvent ne pas être en mesure de comprendre une telle notion et donc de fournir une information adéquate à ce sujet. En effet, l'acceptation traditionnelle de fortes inégalités au sein de la famille, au détriment des femmes notamment - et de leur part aussi -, amènerait une réponse biaisée à la question de leur bien-être individuel.

Il devient en tout cas très clair que le modèle de maximisation de l'utilité du consommateur n'est pas applicable dès lors 1) qu'une personne décide pour une autre ce qu'elle consomme, et 2) que l'allocation de ressources au sein de la famille se fait selon d'autres modalités que les transactions de marché (SEN, 1983, p. 14). Le lien entre les comportements de consommation et le bien-être ou l'utilité individuelle en ressort complètement affaibli. Il n'est pas question non 
plus d'accepter l'« approche économique » de la famille tout juste développée par BECKER (1981) qui, selon SEN (1983, p. 16), ne rend pas justice à l'économie :

Les individus sont considérés comme poursuivant inlassablement leurs utilités individuelles, et ce faisant entrent dans des relations commerciales avec des prix implicites, engendrant les mariages et le travail des familles. Si les utilités individuelles peuvent inclure le souci des autres [...], le processus de maximisation de l'utilité se perpétue sans compromis - sans contraintes ou propriétés, normes ou conventions.

Cette conception lui semble bien trop simple pour saisir la complexité de la relation familiale. Dans tous les cas, son étude de la situation des femmes l'amène à développer un argument supplémentaire - par rapport à ceux qu'il avait mis en avant dans sa critique du welfarisme (SEN, 1977, 1979) ${ }^{4}$ - contre la vision du bien-être en termes d'utilité. Cet argument concerne le biais dans la perception de ses besoins de la part de nombreuses femmes, apparu par exemple sous forme d'une sous-estimation de leurs besoins alimentaires (KYNCH et SEN, 1983, p. 366) ou d'une sous-estimation de leur morbidité (SEN, 1984a, p. 356). En outre, l'identité familiale peut exercer une influence très forte sur la perception qu'une personne peut avoir de son propre bien-être. Par exemple, lorsqu'on interroge une femme indienne du milieu paysan à propos de son bien-être, il se peut qu'elle ne comprenne même pas la question ou qu'elle y réponde en donnant plutôt son opinion sur le bien-être de sa famille (SEN, 1990, p. 131). Cette observation amène SEN (Ibid. pp. 131-133) à formuler quatre propositions fondamentales que nous résumons ci-dessous :

1) il existe des variations considérables dans la perception de l'individualité, mais l'absence de perception du bien-être personnel, là où elle existe, n'est pas immuable ;

2) l'absence de perception de l'intérêt personnel associée à un grand souci du bien-être familial contribue à perpétuer les inégalités traditionnelles ;

3) il existe des aspects objectifs de l'intérêt et du bien-être personnel qui exigent une attention particulière, notamment quand les perceptions individuelles de ces concepts sont biaisées ou inexistantes ;

4) il se peut qu'une personne poursuive d'autres objectifs que son propre bien-être, ce qui est en lien avec sa qualité d'«agent », également pertinente pour l'évaluation de sa situation.

Si l'on s'attache à l'utilité, entendue comme une mesure mentale du bonheur ou de la satisfaction, pour évaluer le bien-être d'une personne, les nombreux problèmes de perception rendront les évaluations peu représentatives de la réalité. En effet, les graves inégalités persistent généralement en raison de leur

4 - Afin de montrer le caractère inapproprié du welfarisme, SEN (1977) a adopté une grille de lecture mettant en avant ses «contraintes informationnelles", c'est-à-dire les informations admises, mais surtout les informations typiquement exclues de l'évaluation. Sans entrer dans le détail de l'argumentation, précisons que pour SEN traiter le bien-être social exclusivement comme une fonction de vecteurs de bien-être individuels restreint considérablement la portée du welfarisme - d'autant plus que l'information sur le bien-être est elle-même souvent très pauvre ou difficile à obtenir. 
acceptation par les victimes elles-mêmes : «l'opprimé finit par accepter cet ordre inégal pour se transformer en un complice implicite» (SEN, 1990, p. 132). C'est par exemple le cas des femmes indiennes de familles pauvres qui se sacrifient pour donner plus de nourriture et de soin aux hommes de la famille, éduquant leurs filles en ce sens également. L'absence de protestation et de remise en question de l'inégalité ne doit pas être, pour SEN (Ibid.), une preuve d'absence de cette inégalité : «il est nécessaire d'aller au-delà des impressions primaires qu'une personne peut avoir sur ces questions, impressions qui peuvent être fondées sur l'acceptation aveugle de certaines priorités traditionnelles ».

Ainsi, ses travaux sur la nature, les causes et les mécanismes de l'inégalité sexuée ont influencé sa compréhension de l'inégalité et de la privation en général et ont conforté sa position théorique vis-à-vis du welfarisme :

Mon scepticisme quant au fait de faire reposer les jugements moraux ou les évaluations politiques sur l'utilité ou sur les préférences [...] a été fortement influencé par ce que j'ai appris des études sur les inégalités de genre, particulièrement concernant le rôle des attitudes et des préférences adaptatives dans la persistance sociale de ces inégalités. (AGARWAL, HuMPHRIES et ROBEYNS, 2005, p. 351)

La notion de «préférences adaptatives » est aujourd'hui bien connue, mais au début des années 1980 peu d'économistes y faisaient référence. SEN lui-même n'employait alors pas cette terminologie, parlant plus $\mathrm{d}^{\prime}$ 'acceptation » ou d'« ajustement» :

L'opprimé apprend si bien à supporter son fardeau qu'il ou elle finit par le négliger. Le mécontentement est remplacé par l'acceptation, la révolte sans espoir par le conformisme silencieux, $[\ldots]$ la souffrance et la colère par une endurance joyeuse. Comme les gens apprennent à s'ajuster aux horreurs existantes par l'absolue nécessité d'une survie sans surprise, les horreurs ont l'air moins terribles dans le système de mesure des utilités. (SEN, 1984b, p. 309)

Dans le contexte spécifique de la discrimination sexuée intrafamiliale, SEN avait abouti à la conclusion que les catégories mentales que représente le plaisir ou le désir sont trop malléables pour servir d'étalon quand on veut mesurer des désavantages ou des privations. À partir de là, il a construit une conception générale de l'adaptation, montrant la nécessité d'une nouvelle grille de lecture en économie. En ce qui concerne la négligence spécifique des préférences adaptatives des femmes, SEN insiste en outre sur la nécessaire remise en cause d'une idée plus générale mais qui affecte aussi les économistes : l'idée «tellement louée, idéalisée, et idolâtrée " de la femme se sacrifiant pour les autres (KAPUR, 1999, p. 3). Cette idée, à son sens, ne sert pas beaucoup les intérêts des femmes, mais sert plutôt à justifier l'ordre établi. C'est donc en ce sens que SEN travaille à mettre en lumière à la fois la position systématiquement inférieure de la femme dans la plupart des sociétés et l'incapacité de la théorie économique telle qu'elle se pratique à évaluer cette inégalité.

Par exemple, il s'attache à montrer que la prospérité d'un ménage dépend grandement de la manière dont les activités extérieures et domestiques s'entretiennent et se soutiennent mutuellement, ce qui revient traditionnellement à une division du travail sexuée. Plus précisément, SEN (1990, p. 130 et 1989, p. 62) 
développe l'idée que les membres d'un ménage sont confrontés simultanément à deux types de problèmes, concernant l'un la coopération - ajouter aux disponibilités totales-, l'autre le conflit - répartir les disponibilités totales. Ces problèmes peuvent être appréhendés par les questions « qui fait quoi ? », « qui peut consommer quoi ? ", et « qui prend quelles décisions ?» (SEN, 1990, p. 130). Or, le fait que seules les activités extérieures - généralement affectées aux hommes - bénéficient d'une reconnaissance sociale et familiale crée des distorsions systématiques dans la perception de qui «produit» quoi et qui «gagne» quoi. La nécessité de prendre en compte les inégalités de genre en économie est donc pertinente à la fois sur le plan de la justice et sur celui de l'efficacité.

\section{III - LA CONCEPTION SENIENNE DE L'AVANTAGE : COMPLÉMENTARITÉ ENTRE LIBERTÉ NÉGATIVE ET LIBERTÉ POSITIVE}

Cette réflexion de SEN, à partir d'observations empiriques des inégalités intrafamiliales, l'amène à intégrer le rôle de la famille en tant qu'institution dans son cadre théorique, en considérant à la fois les aspects conflictuels et coopératifs des relations interpersonnelles en son sein. Le fait d'envisager au contraire ces relations d'une manière non institutionnelle entraînerait certes «des conclusions politiques technocratiques qui seraient directes et sans conflit » (SEN, 1989, p. 61), mais ne permettrait pas de saisir et d'influencer « la nature de la division du travail et des biens qui détermine des répartitions spécifiques des avantages et des caractéristiques particulières d'inégalité» (Ibid., p. 62). L'institution familiale fonctionne très différemment de l'institution marchande, que ce soit au niveau des opportunités de bénéfice ou des conflits d'intérêt. Mais l'analyse des paramètres institutionnels de l'organisation économique et sociale doit, pour SEN, éviter les hypothèses de pure harmonie ou de pur conflit, ce qui implique aussi de s'éloigner d'une caractérisation du comportement individuel en termes de maximisation de l'utilité personnelle. SEN (1989, p. 65, nous soulignons) estime à cet égard que le recours aux analyses des trois penseurs très distincts que sont KANT, SMITH et MARX peut aider à formuler une approche alternative plus compréhensive :

L'analyse de KANT des impératifs catégoriques est, bien sûr, principalement éthique. [...] Dans le contexte présent, la pertinence de ce concept moral repose dans le fait que l'action d'une personne peut être influencée par des considérations éthiques, en plus des considérations matérielles. [...]

La seconde piste vient de la discussion d'Adam SMiTH sur le rôle que les "règles de conduite » jouent en sauvant la société de ce que SMITH appelle la «fausse représentation de l'amour de soi ». [...] nous acceptons certaines règles de comportement, et n'y dérogeons pas même lorsque l'on perçoit un avantage immédiat à cette dérogation. [...]

La troisième piste concerne l'analyse de MARX quant à la nature de l'intérêt personnel et de la perception. Dans cette analyse notre propre perception de notre intérêt personnel est en fait une perception "socialement déterminée ". [...] 
La référence à ces auteurs permet à SEN d'introduire et légitimer trois idées qu'il considère cruciales pour comprendre la nature de la répartition des avantages au sein de l'institution familiale : 1) la notion de «devoir» influence, sans aller jusqu'à le déterminer complètement, le comportement individuel ; 2) des « règles de conduite » peuvent dicter aux individus la manière dont il est convenable ou approprié d'agir ; et 3) l'individu peut avoir une « fausse conscience » de son intérêt propre, liée à son sens de l'identité. Tous ces éléments mis ensemble peuvent expliquer la persistance du désavantage relatif des femmes : « Le rôle que les perceptions non individualistes et d'auto-privation jouent dans l'exploitation des femmes indiennes doit être mieux compris afin d'évaluer la survie et la force des inégalités intrafamiliales dans l'Inde rurale» (Ibid., p. 69). Ces perceptions et les normes de conduite qui en découlent sont certes des «instruments pour la résolution des conflits » latents de l'organisation familiale, mais elles représentent en même temps «les mécanismes de légitimation et de préservation des résolutions inégales de conflits d'intérêt » (Ibid., p. 72). Les perceptions subjectives font donc partie des déterminants objectifs des comportements au sein des familles et de leurs conséquences sur le bien-être ou l'avantage de chacun des membres.

SEN (1983, p. 19) précise à cet égard que les capabilités des personnes offrent « une perspective de la «liberté » dans un sens positif : qui peut faire quoi, plutôt que qui a tel panier de biens, ou qui obtient telle utilité ». Dès lors, «le bien-être familial peut être perçu comme une fonction des libertés positives dont peuvent jouir les différents membres» (Ibid.). Ce sens «positif» de la liberté est une manière de s'éloigner de la conception libertarienne en termes de « droits ». Son étude de la famine avait déjà montré les limites des droits formels ou légaux pour l'accès effectif de certaines catégories de la population à des fonctionnements basiques. Son analyse des inégalités sexuées dans la répartition intradomestique renforce encore sa critique, puisque les droits ne permettent pas de rendre compte des «prétentions légitimes » (SEN, 1990, p. 137) de chacun des membres d'un ménage - prétentions qui dépendent notamment des perceptions de l'individualité et du bien-être que chacun a de soi-même et des contributions perçues à l'aisance économique de la famille (Ibid., p. 141).

SEN (Ibid.) envisage aussi la possibilité d'une «carte élargie des droits à l'échange» qui mettrait en relation une matrice des ressources familiales et un ensemble de matrices de droits familiaux. Cette notion permettrait de mettre en évidence le défaut de droits d'accès effectifs de certains membres - généralement les femmes et les filles - à divers fonctionnements et donc capabilités. Mais une optique purement déontologique permettrait d'observer seulement ce que SEN (1982, p. 37) a appelé, dans une discussion de philosophie morale, les «libertés négatives ». Elle ne suffirait pas à saisir le défaut de puissance d'agir, ou de capacité d'initiative dans le monde social, en particulier pour les plus démunis.

La distinction entre les libertés négatives et les libertés positives n'est pas une invention de SEN. Il s'inspire largement des deux conceptions de la liberté proposées par I. BERLIN (1969). La liberté négative constitue, pour BERLIN (1969, p. 171), «l'espace à l'intérieur duquel un homme peut agir sans que d'autres l'en empêchent». En d'autres termes, l'étendue de cette liberté négative, ou «aire de non-ingérence », dépend de la manière dont autrui le contraint indépendamment 
de ses caractéristiques propres telles que ses capacités intellectuelles et physiques, ses talents et ses handicaps (IGERSHEIM, 2004, p. 98). Par ailleurs, «le sens "positif" du mot liberté découle du désir d'un individu d'être son propre maitre » (BERLIN, 1969, p. 179), ce qui peut se comprendre comme la capacité individuelle «de se libérer de ses propres chaînes : dépendance, ignorance, faiblesse de la volonté, etc. » (IGERSHEIM, 2004, p. 103). L'ouvrage de BERLIN recèle un certain nombre d'ambiguités quant aux interprétations qu'il est possible de faire de ces deux acceptions. Pour sa part, SEN a su développer une interprétation qui fait grand sens avec sa détermination à défendre l'espace des capabilités pour l'évaluation de l'avantage individuel :

Il y a deux manières différentes d'envisager la liberté [...] Une approche voit la liberté en termes "positifs", se concentrant sur ce que chaque personne peut choisir ou réaliser, plutôt que sur l'absence d'un type particulier de restrictions qui l'empêche de faire une chose ou une autre. Par opposition, l'aspect «négatif» de la liberté caractérise précisément l'absence de ce type de contraintes qu'une personne pourrait exercer à l'encontre d'une autre, ou que l'État pourrait exercer sur les individus. Ce contraste, qui a notamment été discuté par Isaiah BerLIN, est important car ces deux manières de caractériser la liberté peuvent aboutir à des traitements très différents. (SEN, 1988, p. 272)

SEN (1991, p. 8, nous soulignons) revient à nouveau sur cette distinction :

la liberté, considérée en termes "positifs », représente ce qu'une personne, toutes choses prises en compte, est capable, ou incapable, d'accomplir. Une telle définition ne prête aucune attention particulière aux facteurs qui expliquent la situation en question [...]. En revanche, la conception «négative» de la liberté met au premier plan l'absence d'entraves à la liberté, entraves qu'un individu [ou l'État ou d'autres institutions] peut imposer à un autre [...].

Ainsi, l'une des conceptions contient l'autre puisqu' une violation de la liberté négative représente aussi un manque de liberté positive, mais l'inverse n'est pas vrai » (Ibid., p. 9). Une démarche d'évaluation complète du point de vue éthique et socialement cohérente doit donc, pour lui, s'intéresser aux deux aspects de la liberté. Le manque de prise en compte de la liberté positive, caractéristique de nombreuses évaluations, explique de nombreuses « horreurs concrètes » (Ibid., p. 6). SEN estime que l'absence de mécontentement ou de désir de changement ne doit pas empêcher de considérer les inégalités. Par exemple, «les femmes de l'Inde rurale n'envient pas, en règle générale, la position des hommes [...] elles n'aspirent pas non plus à une réforme de leur condition » (Ibid., p. 15). Cependant, il faudrait inclure dans la liberté individuelle «la liberté d'évaluer sa propre situation et la possibilité de la changer» (Ibid., p. 16).

Partant, bien que SEN distingue la liberté négative et la liberté positive, il plaide pour leur complémentarité. Sa démarche apparaît alors comme une manière de substituer à la distinction entre liberté positive et liberté négative le concept unique de capabilité, soit l'ensemble des fonctionnements humains qui sont potentiellement accessibles à l'individu. Ce concept soulève deux idées importantes: (1) les capabilités dépendent des caractéristiques personnelles, mais également de l'organisation sociale ; (2) la personne est apte à former des buts qui ne vont pas nécessairement dans le sens de son bien-être. Et contrairement à 
ce que RAWLs a pu objecter, il n'y a pas dans cette philosophie de « conception compréhensive du bien», car la capabilité n'est que «puissance de choix» (CANTO-SPERBER, 1991, p. 35).

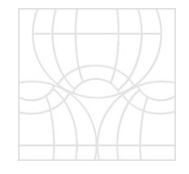

Afin de juger du bien-être des femmes en particulier, il est apparu que l'utilité présentait un sérieux problème lié aux biais de perception que les femmes peuvent avoir envers leurs besoins, leurs intérêts et leur propre bien-être. Ce phénomène aggrave encore les défauts de ce critère - comme la nondifférentiation des sources de satisfaction ou le fondement de l'importance morale des besoins sur la seule notion d'utilité - mis en lumière par la littérature anti-welfariste à laquelle SEN a largement contribué. Étant donné les biais de leurs perceptions subjectives, comparer les niveaux d'utilité entre hommes et femmes ne donnerait pas la mesure de l'étendue des inégalités entre eux.

Parallèlement, les conceptions de l'avantage centrées sur les biens posent, elles aussi, certaines difficultés liées à l'opacité de la répartition intrafamiliale. Il est en effet plus facile d'observer ce qu'une femme peut ou ne peut pas faire, que d'observer les biens auxquels elle a véritablement accès. Il s'agit là encore d'une lacune qui s'ajoute à celles que SEN avait énoncées lors de sa critique des théories ressourcistes, notamment au problème de la variabilité dans la conversion des biens en fonctionnements. D'ailleurs, si l'on pouvait obtenir toute l'information sur les biens et les ressources qu'une femme peut acquérir, en déduire ce qu'elle peut effectivement faire serait difficile (KYNCH et SEN, 1983, p. 366). En outre, SEN (1989) semble supposer que même si l'on pouvait s'assurer d'une répartition égale des biens premiers au sein des familles, encore faudrait-il que les mentalités changent pour qu'hommes et femmes en fassent un usage égal mais cette question sort du cadre d'analyse ressourciste.

Se centrer sur les capabilités apparaît dès lors comme particulièrement légitime dans le cadre de l'étude des inégalités sexuées qui surgissent au sein des familles. À ce niveau, on pourrait se demander à quoi il servirait de mettre en lumière les inégalités si celles-ci existent et persistent en raison de leur acceptation par les victimes elles-mêmes. L'observation d'un défaut de capabilités de base chez une personne revient à faire l'observation d'un aspect objectif de son intérêt personnel, ce qui est distinct de la perception qu'elle a de son propre intérêt. L'idée importante est ici la suivante : «les problèmes de perception ne sont pas immuables» (SEN, 1989, p. 69). C'est en ce sens qu'une meilleure évaluation des inégalités objectives peut amener des changements sensibles de perception, si les résultats sont rendus publics, relayés en politique et dans le système éducatif : «le processus de politisation - notamment la reconnaissance politique de la discrimination sexuelle - peut considérablement modifier ces perceptions, de même que le processus d'évolution économique [ce qui est lié] tels que la participation des femmes au travail à l'extérieur du foyer » (SEN, 1990, p. 132). Dès lors la grille de lecture utilisée pour les évaluations économiques et sociales peut avoir une portée significative. 
Par exemple, le phénomène de la faim ou de la malnutrition constitue pour SEN un défaut de capabilité de base fondamental que les approches resssourcistes et welfaristes ne sont pas aptes à saisir :

Si la lumière est mise sur les privations de capabilités personnelles, alors les inégalités au sein de la famille ont une importance propre, quelle que soit notre vision de la soutenabilité de la notion de bien-être individuel dans des cultures où la famille joue un rôle déterminant. Il n'y a pas d'échappatoire à la tragédie grave que représente la sous-alimentation des petites filles [...], ou la morbidité inhabituelle des femmes. Le problème doit être distingué de la privation des biens en tant que tels, puisque l'approche par les capabilités se préoccupe de ce que les biens peuvent faire aux êtres humains. Partant, la sous-alimentation et la morbidité - pour ne pas mentionner la mortalité fournissent une meilleure représentation que la consommation alimentaire elle-même. (SEN, 1984a, p. 364)

Le défaut de capabilité de base à bien se nourrir peut ainsi être mieux saisi par l'observation des symptômes physiques de la sous-alimentation, comme un poids et une taille anormalement petits ou une morbidité importante, que par l'observation de la consommation alimentaire elle-même. En ce sens, Sen affirme que « le problème de la malnutrition et de la faim ne peut plus être perçu comme une simple question de droits d'accès de la famille»-comme il semblait le postuler dans son étude des famines -, il requiert « une analyse de la division des droits d'accès au sein de la famille » (Ibid.). Ces droits d'accès élargis n'ont plus rien à voir avec des droits légaux, mais sont relatifs aux valeurs et aux normes sociales. Cependant, ce qui doit intéresser l'évaluateur, ce n'est pas tant qui consomme quoi, mais les capabilités dont jouissent les personnes :

l'essence de l'approche par les capabilités est de concevoir la consommation de biens comme rien de plus qu'un moyen de produire des capabilités, et si les capabilités et leur usage peuvent être directement constatés, l'absence d'information détaillée sur la consommation de biens ne doit pas être regrettée. (SEN, 1984c, p. 522)

Toutefois, dans certains cas - quand la capabilité est difficilement observable -, « il peut y avoir un avantage pratique à utiliser des données ajustées ${ }^{5}$ sur les biens en tant que données indirectes sur les capabilités » (Ibid., p. 523). Il se peut aussi que les droits d'accès soient suffisamment déterminants des capabilités individuelles pour être observés. SEN (Ibid.) considère que c'est par exemple le cas pour le problème extrême de la famine, donnant ainsi une légitimité à son ouvrage de 1981. En revanche, pour les problèmes moins extrêmes de malnutrition endémique, de morbidité et de mortalité élevées, «il est important de se rappeler que les droits d'accès ne représentent rien de plus qu'une partie de l'histoire»(Ibid.). L'image de la société - et des avantages individuels - que donne la description des droits d'accès est donc incomplète ; pire, elle peut être la source de l'échec des politiques publiques visant à lutter contre les privations comme l'atteste son étude de l'effet des réformes agraires sur la situation des femmes (SEN et SENGUPTA, 1983).

5 - SEN ne précise pas ici ce qu'il entend par données « ajustées », mais on peut supposer qu'il s'agit d'envisager la consommation des biens en fonction de ce qu'ils peuvent permettre comme fonctionnement individuel. Par exemple, les données sur la consommation alimentaire doivent être en lien avec les caractéristiques nutritives des aliments consommés et les besoins de l'individu qui consomme - l'intérêt de ces données reposant principalement sur leurs effets nutritionnels et leurs conséquences en termes de capabilités. 
En fait, ses écrits laissent apparaître une certaine ambiguïté quant au choix de la dimension individuelle pour l'évaluation. Malgré l'accent indéniable sur les capabilités, les difficultés d'observation de cette dimension l'amènent souvent à se rabattre sur les fonctionnements effectifs. Il justifie à plusieurs reprises ce rétrécissement de l'analyse, principalement en arguant du fait que, concernant les capabilités de base, il y a peu de chance qu'un individu ne saisisse pas les opportunités dont il dispose. Son argument peut sembler à première vue valable, en particulier lorsqu'on s'intéresse à la capabilité à bien se nourrir. Cependant, les choses se compliquent dès lors que certains individus choisissent de jeûner volontairement, pour des raisons religieuses ou autres. Or, ces choix peuvent refléter la «qualité d'agent» [agency] d'une personne, ce que SEN considère comme plus pertinent que le bien-être pour fonder les évaluations des états individuels et sociaux.

Il existe certes des liens forts entre «bien-être » et «qualité d'agent», d'une part parce que le bien-être d'une personne peut conditionner la réalisation de ses autres objectifs et, d'autre part parce que la qualité d'agent d'une personne peut augmenter son niveau de bien-être. Mais les deux notions ne doivent pas être identifiées, et l'évaluation des capabilités est plus à même de refléter la qualité d'agent que l'évaluation en termes d'utilité, ou même de fonctionnement. La difficulté, c'est que «l'aspect «agent » d'une personne, est certainement celui qui est le plus influencé par son sens des obligations et sa perception d'un comportement légitime»(SEN, 1990, p. 127). Tout comme l'utilité est une notion très malléable - en raison des problèmes de perception et des préférences adaptatives -, le choix d'une personne parmi les fonctionnements qui lui sont accessibles est plus ou moins conditionné par les normes sociales et les perceptions conventionnelles de légitimité. D'où l'importance de tenir compte, au-delà des capabilités d'accomplir différentes choses, de la capabilité de pouvoir choisir parmi les fonctionnements accessibles.

\section{BIBLIOGRAPHIE}

Agarwal B., Humphries J. et Robeyns I., 2005, «Continuing the Conversation. Amartya Sen talks with Bina Agarwal, Jane Humphries and Ingrid Robeyns ", in AgARWAL B., Humphries J. et ROBEYNS I., (eds.), Amartya Sen's Work and Ideas: A Gender Perspective, London/ New York, Routledge, pp. 321-338.

Becker G. S., 1981, A Treatise on the Family, Cambridge (Massachusetts), Harvard University Press.

BERLIN I., 1969, Four Essays On liberty, Oxford, Oxford University Press.
Canto-Sperber M., 1991, « Choix de Vie et Liberté. Sur l'œuvre d'Amartya Sen », Esprit, mars-avril, pp. 26-38.

Chen L. C., HuQ E., D’Souza S., 1980, «A Study of Sex-Biased Behavior in the Intra-Family Allocation of Food and the Utilization of Health Care Services in Rural Bangladesh », International Centre for Diarrhoeal Disease Research, Bangladesh and Department of Population Sciences, Harvard School of Public Health. 
CMDA, 1980, « Health and Socioeconomic Survey of Calcutta Metropolitan Development Area », Calcutta, I.S.I. and Calcutta Metropolitan Development Authority.

FAO/WHO Expert Committee, 1973, Energy and Protein Requirements, Rome, Food and Agricultural Organisation of the United Nations.

IGERSHEIM H., 2004, Liberté et Choix Social. Contribution à l'analyse de la liberté en économie normative, thèse de Doctorat es Sciences Économiques, Université Louis Pasteur, Strasbourg I, 10 décembre, 435 pages.

INFS, 1977, Nutrition Survey of Rural Bangladesh 1975-76, Dacca, University of Dacca.

KAPUR A, 1999, «Amartya Sen: Human Development - Interview ", The Atlantic Unbound, December, 15, http:// www.theatlantic.com/unbound/ interviews/ba991215.htm.

KYNCH J., SEN A. K., 1983, "Indian Women: Well-being and Survival», Cambridge Journal of Economics, Vol. 7, n³/4, Sept.-Dec., pp. 363-380.

PadmanabHa P., 1981, «Provisional Population Totals ", Series-1 India, Paper-1, Census of India 1981, New Delhi, Office of the Registrar General of India.

SEN A. K., 1977b, "On Weights and Measures: Informational Constraints in Social Welfare Analysis ", Econometrica, Vol. 45, n 7, Oct., pp. 1539-1572.

SEN A. K., 1979, «Utilitarianism and Welfarism », Journal of Philosophy, Vol. 76, n 9, pp. 463-489.

SEN A. K., 1981, Poverty and Famines: An Essay on Entitlement and Depravation, Oxford, Clarendon Press.

SEN A. K., 1982, "Rights and Agency », Philosophy and Public Affairs, Vol. 11, $\mathrm{n}^{\circ}$ 1, Winter, pp. 3-39.
SEN A. K., 1983, "Economics and the Family", Asian Development Review, Vol. 1, n 2, pp. 14-26.

Sen A. K., 1984a, « Family and Food: Sex Bias in Poverty », in SEN A. K., 1984, Resources, Values and Development, Oxford, Blackwell et Cambridge, (Massachusetts), Harvard University Press, pp. 346-368.

Sen A. K., 1984b, «Rights and Capabilities ", in SEN A. K., 1984, Resources, Values and Development, Oxford, Blackwell et Cambridge (Mass.), Harvard University Press, pp. 307-325.

Sen A. K., 1984c, "Good and People», in SEN A. K., 1984, Resources, Values and Development, Oxford, Blackwell et Cambridge, Massachusetts, Harvard University Press, pp. 509-532.

SEN A. K., 1987, «The Standard of Living: Lecture II, Lives and Capabilities ", in G. HawTHORN, The Standard of Living, Cambridge, Cambridge University Press, pp. 20-38.

SEN A. K., 1988, "Freedom of Choice: Concept and Content », European Economic Review, Vol. 32, $\mathrm{n}^{\circ}$ 1-2, pp. 269-294.

SEN A. K., 1989, " Cooperation, Inequality, and the Family ", Population and Development Review, Vol. 15, Supplement: Rural Development and Population: Institutions and Policy, pp. 61-76.

SEN A. K., 1990, «Gender and Cooperative Conflicts ", in Tinker I., (ed.), Persistent Inequalities, New York, Oxford University Press, pp. 123-149.

SEN A. K., 1991, «La Liberté Individuelle: Une Responsabilité Sociale», Esprit, mars-avril, pp. 5-25.

Sen A. K., Sengupta S., 1983, « Malnutrition of Rural Indian Children and the Sex Bias", Economic and Political Weekly, n 18, May, pp. 855-864. 